\title{
A Multi-Objective and Evolutionary Hyper-Heuristic Applied to the Integration and Test Order Problem
}

\author{
Giovani Guizzo*, Silvia R. Vergilio, Aurora T. R. Pozo, Gian M. Fritsche \\ DInf - Federal University of Parana, CP: 19081, CEP 19031-970, Curitiba, Brazil
}

\begin{abstract}
The field of Search-Based Software Engineering (SBSE) has widely utilized Multi-Objective Evolutionary Algorithms (MOEAs) to solve complex software engineering problems. However, the use of such algorithms can be a hard task for the software engineer, mainly due to the significant range of parameter and algorithm choices. To help in this task, the use of Hyper-heuristics is recommended. Hyper-heuristics can select or generate low-level heuristics while optimization algorithms are executed, and thus can be generically applied. Despite their benefits, we find only a few works using hyper-heuristics in the SBSE field. Considering this fact, we describe HITO, a Hyper-heuristic for the Integration and Test Order problem, to adaptively select search operators while MOEAs are executed using one of the selection methods: Choice Function and Multi-Armed Bandit. The experimental results show that HITO can outperform the traditional MOEAs NSGA-II and MOEA/DD. HITO is also a generic algorithm, since the user does not need to select crossover and mutation operators, nor adjust their parameters.
\end{abstract}

Keywords: Metaheuristic, Hyper-heuristic, multi-objective algorithm, search-based software engineering, software testing

\footnotetext{
${ }^{*}$ Corresponding author

Email addresses: giovaniguizzo@gmail.com (Giovani Guizzo), silvia@inf.ufpr.br (Silvia R. Vergilio), aurora@inf.ufpr.br (Aurora T. R. Pozo), gianfritsche@gmail.com (Gian M. Fritsche)
}

Preprint submitted to Applied Soft Computing

March 13, 2017 


\section{Introduction}

Search-Based algorithms have been successfully applied to solve hard software engineering problems in the field of Search-Based Software Engineering (SBSE) [23]. Some of the most used algorithms in SBSE are Multi-Objective 5 Evolutionary Algorithms (MOEAs). Such algorithms are based on Pareto dominance concepts and offer to the user a set of good solutions that represent the best trade-off between different objectives, which, in software engineering, are generally associated to software metrics to evaluate the quality of a solution.

However, the usage of a MOEA is not always easy for the software engineer. Many times, it demands effort to adapt implementation details, to adjust parameters (e.g. mutation and crossover probabilities), to select search-based operators, and so on. Another difficulty is that some of them are adjusted, designed or evaluated for a specific problem, which can affect their generality [8].

To overcome these limitations, the hyper-heuristic field has emerged. A hyper-heuristic is a methodology to automate the design and tuning of heuristic methods to solve hard computational search problems [9]. It is used to select or generate new low-level heuristics (LLHs) while algorithms are being executed. The idea is to provide more generally applicable and flexible algorithms, while yielding better results than conventional algorithms alone. A distinctive characteristic of hyper-heuristics is that they operate over the heuristic space instead of the solution space. This can be interpreted as a technique to select or generate the best heuristic that solves the problem, instead of solving the problem directly [8]. To allow this, a hyper-heuristic dynamically guides the search process by managing a set of LLHs (e.g. metaheuristics and genetic operators).

25 The usage of such methodology in SBSE has raised interest. Harman et al. [22] state that hyper-heuristics can contribute to obtain holistic and generic SBSE algorithms. However, we can find few works applying hyper-heuristic in SBSE [6, 27, 31, 37]. Motivated by this, we introduced HITO in [21], a Hyper-Heuristic for the Integration and Test Order Problem.

The Integration and Test Order Problem (ITO) consists in generating an 
order for the units (the smallest part of a software) to be integrated and tested, in a way that the stubbing cost is minimized [4]. A stub is an emulation of a unit that is not yet implemented, tested or integrated in the software. Therefore, a unit that is required by another unit shall be emulated to enable a proper testing 35 or integration. The underlying cost of this problem is the potentially great number of stubs that may be generated during the testing activity. These stubs will be obsolete once the emulated units are implemented, and consequently, such stubs become wasted resources. By rearranging the unit ordering, we can force the most required units to be integrated and tested first, so that the next units will not require a stub for such a unit. This problem has some characteristics that make it suitable for application of hyper-heuristics [21]. The cost is impacted by different factors, such as size/complexity/number of classes, methods, attributes, return types, parameter types, and any other element that must be emulated in order to proceed with the integration and testing. Hence multi-objective algorithms have presented good results [4]. It is possible to use several search operators and the problem is found in several contexts such as object- and aspect-oriented testing, thus, it may be configured in several ways.

To allow a proper, generic and efficient solution to the ITO problem, HITO uses a selection method and a novel rewarding measure to select the best LLH 5o (combination of crossover and mutation operators) at each evolutionary mating. By using HITO the software engineer does not need to select the crossover and mutation operators to be used by the MOEAs. He/she does not need to choose the probabilities for these operators.

HITO was designed to be flexible and robust, hence it has the following main characteristics: i) a set of steps to select and apply LLHs using MOEAs; ii) a set of parameters to allow the user to personalize its functionality; iii) selection of the best LLH at each mating, in contrast to other works (such as [28, 31, 36]) that perform the selection at each generation; iv) a rewarding function that is based on the Pareto dominance concept and uses solely the parents and children 6o involved in the mating, rather than using quality indicators such as in [36]; v) score based selection methods, e.g. Choice Function (CF) [36] and Multi-Armed 
Bandit (MAB) [19], which can also be chosen by the user. We acknowledge that these characteristics can help HITO in overcoming the limitations of MOEAs and also obtaining the best results. Preliminary results [21] proved that HITO 65 is capable of solving real instances of the ITO problem.

Considering the HITO advantages and its promising results, this work extends [21] by providing a better description and a deep analysis of HITO with new experimental results. We can mention the following contributions:

- We propose an improved reward measure that was redesigned to encompass different numbers of parents and children involved in the mating (e.g. generating one child or generating two children);

- We present results from a broader evaluation encompassing two quality indicators, two statistical tests, a set of many objective functions, three versions of HITO (using MAB, CF and a random selection strategy), and two conventional MOEAS: i) NSGA-II: well-known and widely applied in SBSE.; and ii) MOEA/DD: state of the art algorithm for multi-objective problems. This new empirical evaluation was conducted to improve the accuracy of the evaluation of HITO, and to assess not only how well it performs when compared to conventional algorithms, but also to assess if a random LLH selection is enough for this problem;

- We use four different objective functions to analyze how a selection hyperheuristic such as HITO behaves with many objectives. The experimentation shows favorable results for all considered systems.

In the experimentation we used two sets of objective functions to evaluate 85 the solutions: a set based on two metrics (number of attributes and number of methods to be emulated), and a set based on four metrics (number of attributes, number of methods, number of return types, and number of parameter types to be emulated). These are the same objective functions used in previous works $[4,21,37]$. Furthermore, we applied the algorithms on 7 real world эo object-oriented and aspect-oriented systems. These are well known and widely 
used systems $[4,3,21,47,37,48]$, with different characteristics and varying in size (lines of code, number of classes, etc). The results are favorable for HITO in terms of quality indicators and statistical analysis. HITO was able, in overall, to outperform all the MOEAs with statistical significance, while being outperformed by MOEA/DD in only one system.

This work is organized as follows: Section 2 contains a brief explanation of hyper-heuristics, their main components and the selection methods used in this work. Section 3 reviews the ITO problem. Section 4 presents related work; Section 5 presents a detailed description of HITO, the reward measure being proposed, the LLHs, the credit assignment measures and the adapted selection methods implemented in this work. Section 6 describes and analyses empirical results from HITO evaluation. Finally, Section 7 presents the concluding remarks and future works.

\section{Hyper-Heuristics}

Hyper-heuristics are often defined as "heuristics to select or generate heuristics" [9]. A hyper-heuristic may be used to select the most appropriate heuristics or to generate new heuristics using existing ones. Chakhlevitch and Cowling [10] define hyper-heuristics as higher level heuristics that: i) manages a set of LLHs; ii) searches for a good method to solve a problem rather than searching for a good solution; and iii) uses only limited problem-specific information.

The goal is to find the right method or sequence of heuristics to be used in a given situation rather than trying to directly solve the problem [8]. Thereby, one of the main ideas is to develop algorithms that are generally applicable in several problem instances [8]. By achieving that generality, the algorithm may be used with less human effort and additionally to obtain better results. This idea was motivated by the difficulties regarding the application of conventional search techniques, such as the great number of parameters for configuring algorithms and the lack of guidance on how to select the right LLHs [8].

It is important to emphasize the "limited problem-specific information" used 
by the hyper-heuristic approaches [10]. The idea is to maintain a "domain barrier" that channelizes and filters the domain information visible by the hyperheuristics. In other words, the hyper-heuristic should be independent of the problem domain by only having access to some domain-independent information from the domain barrier $[9,10]$.

In this paper we use the hyper-heuristic definition given by Burke et al. [9]: "A hyper-heuristic is an automated methodology for selecting or generating heuristics to solve hard computational search problems". The authors also proposed a classification for hyper-heuristics as seen in Figure 1. We can see two main dimensions [9]: i) the heuristic search space nature; and ii) the sources of heuristic feedback. The heuristic search space nature defines if a hyper-heuristic is either used to: i) select existing LLHs; or ii) generate LLHs using components of existing ones. In this categorization there is a second level dimension that is concerned with the nature of the LLHs used by the hyper-heuristic: i) construction; or ii) perturbation. The construction heuristics start with an empty solution, and gradually build a complete solution. On the other hand, the perturbation heuristics start with a complete solution (either randomly generated or gradually built by construction heuristics) and try to iteratively improve it.

Figure 1: Classification of hyper-heuristic approaches according to [9]. Extracted from [9].

The second dimension is the source of heuristic feedback [9]. The learning hyper-heuristics can be divided into two categories: i) offline learning; and ii) 140 online learning. Offline learning hyper-heuristics gather knowledge from a set of training instances, and use this knowledge to generalize the solving of unseen instances. Online hyper-heuristics use online information about the performance of LLHs to dynamically select them, thus it is potentially more flexible than offline approaches. There are also non-learning hyper-heuristics (e.g. random) that simply do not use feedback to guide the search.

In this paper we work with a hyper-heuristic for online selection of perturbation LLHs, more specifically for selecting search operators for Multi-Objective 
Evolutionary Algorithms (MOEAs) [11]. Selection hyper-heuristics use two main components [8]: i) heuristic selection method; and ii) move acceptance method. In addition, each of these methods can vary independently, which brings more generality to the hyper-heuristic approaches. A selection method uses techniques to select a LLH in a given moment of the search, either using a learning or a non-learning approach, whereas the move acceptance method decides whether a solution obtained by the selected LLH must be accepted.

The hyper-heuristic presented here uses two score-based heuristic selection methods: Choice Function (CF) [12] and Multi-Armed Bandit (MAB) [19].

\subsection{Choice Function}

The Choice Function (CF) adaptively ranks the LLHs according to their previous performances [12]. CF was proposed initially to select LLHs based on their scores and using several strategies in order to solve the Scheduling Sales Summit combinatorial problem. The promising results of the authors motivated other works to use $\mathrm{CF}$ as a selection method, such as $[29,36]$.

The credit assignment equation of $\mathrm{CF}$ is formulated according to the works of Cowling et al. [12] and Kendall et al. [29], and was presented as follows:

$$
f\left(h_{i}\right)=\alpha f_{1}\left(h_{i}\right)+\beta f_{2}\left(h_{i}, h_{j}\right)+\delta f_{3}\left(h_{i}\right)
$$

where $h_{i}$ is the LLH being evaluated; $h_{j}$ is the LLH that has just been applied; $f_{1}\left(h_{i}\right)$ is the recent improvement of $h_{i} ; f_{2}\left(h_{j}, h_{i}\right)$ is the recent improvement of $h_{i}$ when called immediately after $h_{j} ; f_{3}$ counts the CPU seconds that have passed since $h_{i}$ was last called; and the variables $\alpha, \beta$ and $\delta$ are the weight parameters in the interval $[0,1]$ for the functions $f_{1}, f_{2}$ and $f_{3}$ respectively.

In Equation 1, the functions $f_{1}$ and $f_{2}$ are used for intensification purposes, i.e., for a better exploitation of the search space by favoring the LLHs that have been yielding the best results so far [29]. In contrast, $f_{3}$ is used for the diversification of the solutions, i.e., for a better exploration of the search space by favoring LLHs that have not been applied in a while. These functions and their weight parameters were introduced in the equation to balance the search 
between the intensification and diversification factors. If a greater intensification is desired, then $\alpha$ must be increased, or decreased otherwise.

Equation 2 shows a simplified version of the credit assignment of $\mathrm{CF}$ used in [36]. This version contains only two functions: $f_{1}$ and $f_{2}$.

$$
f(h)=\alpha f_{1}(h)+\beta f_{2}(h)
$$

where $f(h)$ gives a score for a LLH $h ; f_{1}$ reflects the recent improvement of $h$; $f_{2}$ is the elapsed CPU seconds since $h$ has been called; and $\alpha$ and $\beta$ are the weight parameters to balance the values of $f_{1}$ and $f_{2}$.

We use in this work the adaptation of CF proposed by Maashi et al. [36] due to its promising results and easy usage. We propose a different mechanism to evaluate LLH improvements using solely the concept of Pareto dominance as $f_{1}$ and elapsed iterations as $f_{2}$. We adopted these changes in order to design a CF more compatible with HITO.

\subsection{Multi-Armed Bandit}

Auer et al. [5] proposed the Upper Confidence Bound (UCB) selection strategy inspired by the Multi-Armed Bandit (MAB) problem [5, 19, 33]. In this problem there is a set of $K$ independent arms and each arm has an unknown probability of giving a reward. The goal is to maximize the accumulated reward by pulling the arms in an optimal sequence. Other works such as [13, 19, 24, 35] proposed new algorithms to adaptively select operators using the MAB and UCB inspiration. In this work, we call $\mathrm{UCB}$ as $M A B$ method, and the other MAB based selection methods as a derivation of the MAB method.

The idea behind the MAB method is that a given operator $i$ is associated to two main values $[5,19]$ : i) an estimated empirical reward $\hat{q}_{i}$ that measures the empirical reward of the operator over time; and ii) a confidence interval, depending on the number of times the operator was executed. The MAB method selects the operator with the best value according to Equation 3 [19]:

$$
\arg \max _{i \in I}\left(\hat{q}_{i}+C \sqrt{\frac{2 \log n}{n_{i}}}\right)
$$


where $i$ is the $i$-th operator of a set of operators $I ; \hat{q}_{i}$ is the average reward obtained by $i$ so far; $C$ is the scaling factor parameter, i.e., controls the tradeoff between the exploration and exploitation; $n$ is the overall number of operator executions so far; and $n_{i}$ is the number of times the operator $i$ was executed.

Originally, the scaling factor $C$ was not introduced in the MAB equation [5], but was proposed by Fialho et al. [18] to balance the scales of exploration and exploitation. If the exploration is desired, then the $C$ parameter must be increased. However, if exploitation is desired, then $C$ must be decreased. In essence, other adaptations of the MAB method vary the computing of $\hat{q}_{i}$ and $n_{i}$ (i.e. credit assignment and memory length), while maintaining the heuristic selection as the max value for Equation 3.

In this paper we use a variation of the original MAB method, which is called Sliding Multi-Armed Bandit (SlMAB) proposed in [19]. This strategy introduces a memory length adjustment and a credit assignment function that allow a faster identification of changes on the performance of LLHs. Because of this, we decided to use SIMAB in this paper.

The SIMAB credit assignment uses two main functions to compute the score of a given operator. Equation 4 shows how SIMAB computes the reward estimate $\hat{q}_{i}$, which corresponds to the exploitation factor:

$$
\hat{q}_{i, t+1}=\hat{q}_{i, t} \frac{W}{W+\left(t-t_{i}\right)}+r_{i, t} \frac{1}{n_{i, t}+1}
$$

where $i$ is the $i$-th operator; $t$ is the current time step (e.g. algorithm iteration or generation); $t_{i}$ is the last time step in which $i$ was applied; $W$ is the size of the sliding window; $r_{i, t}$ is the instant/raw reward given to the operator $i$ at the time step $t$; and $n_{i, t}$ is the number of times $i$ was applied until the time step $t$.

The key element in this equation is the introduction of a sliding window of size $W$, which is the memory length. A sliding window is a type of memory that stores the last $W$ rewards obtained by the operators. These values are used to compute the quality of the operator. This can be done in several ways, but in this work we use the extreme credit assignment, which defines the reward of an operator as the best reward found in the sliding window. We adopted this 
strategy because Fialho et al. [19] concluded that it is the most robust operator selection method in combination with SIMAB.

The second function (described in Equation 5), which composes the credit assignment component of SIMAB, is used to compute the exploration factor.

$$
n_{i, t+1}=n_{i, t}\left(\frac{W}{W+\left(t-t_{i}\right)}+\frac{1}{n_{i, t}+1}\right)
$$

where $n_{i, t}$ is the application frequency of $i$ until the time step $t$; and the other variables are the same as the previous equation.

This equation demands a counter $n_{i, t}$ for storing the application frequency for each operator. This credit assignment is later used in Equation 3, where the operator that maximizes the equation is chosen to be applied.

The good learning capability of MAB and its results in other works [19, 35] are our main motivations for its usage. Furthermore, we expect that this method properly guides the hyper-heuristic search with more accuracy than $\mathrm{CF}$, due to its mechanisms designed for a continuous learning. We adapted the SIMAB implementation in order to make it more compatible with the hyper-heuristic proposed in this paper. The adaptations of CF and SIMAB can be seen in Sections 5.3 and 5.4 respectively.

\section{The Integration and Test Order Problem}

The unit test focuses on testing the smallest part of the program. However,

235 a software usually has several units that must be integrated and tested in order to reveal interaction problems between them. This activity, called integration testing, sometimes requires the creation of stubs. A stub is an emulation of a unit that is created when such unit is required by other units, but it is not yet available. The problem in creating stubs is that the development of these artifacts is error-prone and costly [48]. Therefore, minimizing stubbing costs is fundamental to reduce the testing efforts and costs. This minimization can be done by finding an optimal sequence of software units for integration and testing, which is the problem known as ITO problem. 
The ITO problem is found in different development contexts [4], such as: 245 i) component-based [26]; ii) object-oriented (OO) [4, 48]; iii) aspect-oriented (AO) [4]; and iv) software product lines [25]. Nevertheless, to represent units and their interdependencies, one may use different kinds of models, such as graphs, Test Dependency Graphs (TDG) [45], and Object Relation Diagrams (ORD) [32]. The latter is the most used for OO and AO contexts [48]. An ORD of a system contains its classes, interfaces and their interdependencies modeled as a graph, where the classes and interfaces are the vertices and the dependencies are the edges. The ORD graphs are represented by data matrices that are used by the algorithms to compute the cost of each unit sequence. In this paper we use an extension of ORD (proposed in [4]) to include the AO components in this graph, such as aspects, advices and join points. This allows the execution of algorithms previously used only in $\mathrm{OO}$ context, for $\mathrm{AO}$ systems. In addition, based on the results of [4], we use a combinatorial approach in which classes and aspects are all integrated and tested together.

In other contexts the tester must be concerned about how many dependency cycles he/she must break in order to minimize the test cost. If a cycle is broken, then a stub must be created to fulfill the dependency. When there are no cycles in the dependency graph, then a simple inverse topological sort of the graph can find a solution that does not require stubs [4]. On the other hand, if a dependency graph has many cycles, then the problem becomes complex and search-based algorithms are applicable to find good orders. Some SBSE works have applied metaheuristics to solve this problem $[4,7,47,48]$.

We choose to tackle this problem due to its main characteristics that allow a robust application of hyper-heuristics: i) it is properly solved by multiobjective algorithms [3, 4, 47]; ii) the representation of the problem is the same 270 as permutation problems, which provides several operators to be selected by the hyper-heuristic; iii) the problem can be addressed in several contexts; and iv) it is a real problem, hence if effectively solved, the engineer effort invested in this activity can be significantly reduced. In this sense, the objective of applying hyper-heuristics in this work is to provide a generic and robust approach to solve 
this problem, while also outperforming traditional algorithms (such as Genetic Algorithms - GAs) on the minimization of stubbing cost.

\section{Related Work}

We split this section in two subsections: the first one describes the works that use evolutionary computation to solve the ITO problem, and the second

There is an area related to hyper-heuristics called "Adaptive Operator Selection" (AOS) [38], which aims at adaptively selecting evolutionary operators 
during the optimization process of genetic algorithms. It is our understanding that AOS can be considered a sub-area of hyper-heuristics, but this is not a consensus in the community. We classify our approach as a hyper-heuristic because it uses concepts and components from the hyper-heuristic literature. Furthermore, a hyper-heuristic can be applied to a wider set of different heuristics such as optimization meta-heuristics, and not only to operators. Thus, we believe that the mechanisms used by HITO are better classified in the hyper-heuristic field because they can be easily adapted to other kinds of LLHs.

Some surveys have already cited hyper-heuristics as trends and future research topic for SBSE [22, 41]. However, very few works explore the usage of hyper-heuristics for solving software engineering problems.

Kumari et al. [31] proposed a multi-objective algorithm called Fast Multiobjective Hyper-heuristic Genetic Algorithm (MHypGA) to solve the module clustering problem. The proposed hyper-heuristic selects LLHs while the optimization is being executed. Each LLH is composed by a selection operator, a mutation operator and a crossover operator. The authors empirically evaluated MHypGA with six real-world problems. MHypGA outperformed a conventional evolutionary algorithm in all problems.

Basgalupp et al. [6] applied an offline hyper-heuristic to evolve an algorithm for the generation of effort-prediction decision trees. The authors concluded that the algorithm created by their hyper-heuristic was able to obtain better results than some state-of-the-art algorithms and other traditional heuristics.

Jia et al. [27] proposed a single online hyper-heuristic algorithm to intelligently learn and apply combinatorial interaction testing strategies. The goal of the authors is to obtain better solutions and to provide an algorithm more generally applicable. The experimental evaluation compares the results of their hyper-heuristic approach to the results of state-of-the-art techniques and to the best known results of the literature. The hyper-heuristic performed well on constrained and unconstrained problems in several instances.

Kateb et al. [28] propose Sputnik, a hyper-heuristic designed to online select mutation operators for MOEAs in order to improve the performance and 
reduce the cost of cloud infrastructures. Sputnik uses a score-based selection mechanism and apply the best mutation operator for a whole generation before evaluating and selecting the operators again. In the experiments, the authors observed that Sputnik achieved better trade-offs than a Random algorithm, and also decreased the number of generations needed to obtain acceptable results.

In a previous work [37], we introduced an offline hyper-heuristic to generate MOEAs for solving the ITO problem. The drawback of this hyper-heuristic is its great computational cost in generating the LLHs and its low flexibility when compared to online hyper-heuristics such as HITO. As far as we are aware, this is the only hyper-heuristic besides HITO for this kind of problem, and one of the few hyper-heuristics ever applied to software testing.

We treat the ITO problem as multi-objective in order to increase the accuracy in measuring the real effort needed to develop the stubs. In the literature, some works such as $[1,20,28,31,36,39,42,43,44,46]$ proposed multi-objective hyper-heuristics using evolutionary algorithms and LLH selection. However, most of these works are concerned in selecting construction LLHs, meta-heuristics, heuristics to generate new heuristics, or using offline approaches to train the algorithms. To the best of our knowledge, only [28, 31] use online hyper-heuristics for selecting perturbation LLHs, such as mutation and crossover operators, using genetic algorithms. We recommend [8], a complete survey on hyper-heuristics, for a more detailed description on the hyper355 heuristics applicability in evolutionary computation.

\section{HITO}

Hyper-heuristic for the Integration and Test Order Problem (HITO) is an online hyper-heuristic for selecting perturbative LLHs (mutation and crossover operators) used by MOEAs in order to solve the ITO problem. The main goal of HITO is to select in each mating the best combination of mutation and crossover operators based on their previous performances. Our hyper-heuristic does not wait for one or more generations to select a LLH (such as in $[31,36]$ ), 
but rather keeps the scores of LLHs updated and selects the best one in each mating (parent combination for generating children). HITO is designed to be generic and used by any MOEA, therefore the user must provide five inputs that are used in the HITO workflow. Next and taking Figure 2 as basis, we better explain how HITO works, its main steps and its main components.

Figure 2: HITO workflow

In the first step of HITO, Initialize Configuration, the hyper-heuristic is initialized using the some inputs given by the user: i) the instance of the problem to be optimized; ii) the fitness functions to evaluate the solutions; iii) a set of LLHs (combination of a mutation and a crossover operator) to generate solutions compatible with the problem representation; iv) a MOEA and its parameters to perform the optimization using the fitness functions and the LLHs; and v) a selection method to select the best LLH in each mating of the MOEA.

In this work, we use as inputs ORDs of real systems and NSGA-II [14] as the main MOEA of HITO. Differently to our previous work [21], we use as input four different objective functions: 1) Number of Attributes (A): counts the number of attributes to be emulated by the stubs; 2) Number of Operations (O): counts the number of operations (including constructors) to be emulated by the stubs; 3) Number of Distinct Return Types (R): counts the number of distinct return types for the operations of the stubs; 4) Number of Distinct Parameters $(\mathrm{P})$ : counts the number of distinct parameter types for the operations of the stubs.

The objectives are the same ones reported in the literature [7, 4]. Even though these objectives are not necessarily conflicting, they may be optimized simultaneously and independently, i.e., the optimization process may find several non-dominated solutions regarding these four objectives, but it is still a hard task to balance the trade-off. An advantage is to enable the tester to choose from the Pareto front the non-dominated solution that best fits his/her purposes. For instance, if the tester is not comfortable emulating operations, he/she can choose a non-dominated solution with almost no operations to be emulated. If 
the tester is only concerned with return types and parameter types, he/she can choose solutions with lower $\mathrm{R}$ and $\mathrm{P}$ values, and greater $\mathrm{A}$ and $\mathrm{O}$ values.

The problem is represented as an array of units and optimized as a permutation problem. Due to this, we use the LLHs specific for this kind of problem (see Subsection 5.1). Moreover, we also implemented and adapted two selection methods: CF [36] and MAB [19] (see Sections 5.3 and 5.4).

The second step of HITO is Initialize Low-level Heuristics. Each LLH has an associated value for each of the two measures: i) a reward measure, denoted as $r$ in the interval $[0,1]$; and ii) a time measure, denoted as $t$ in the interval ${ }_{400}[0 . .+\infty]$. The value of the reward measure $r$ is the instant reward given to a LLH regarding its performance. The greater the $r$ reward, the better its recent performance. In contrast, the value of the $t$ measure counts how many matings have past since the last application of that LLH. The greater the $t$ value, the longer the LLH has been idle. Both values are initialized as 0 for all LLHs. 405 Subsection 5.2 presents $r$ and $t$ and how they are computed for each LLH.

After initializing the LLHs, in the Initialize Population step, the first population is created by the MOEA according to its own initialization strategy (usually random). This population is then evaluated by the objective functions.

Following, in the Loop of Generations step, generations of solutions are cre410 ated until the stop criterion is met. Firstly, for each generation, the pool (population) of offspring solutions must be filled. The size of this pool varies for each MOEA, but usually it is the population size.

For filling this pool, first two parents must be selected (Select Parents) to generate new children. This selection is specific for each MOEA, e.g., for NSGA415 II the parent selection is done by a binary tournament. After this, a LLH is selected (Select Low-Level Heuristic) using the hyper-heuristic selection method, which in turn uses the LLH values of the measures $r$ and $t$. HITO then applies the crossover and mutation operators provided by the LLH (Apply Low-Level Heuristic) in order to generate offspring solutions from the selected parents.

420 Usually two children are generated, but it may vary for each MOEA. If these new solutions violate any constraint, then a correction algorithm can be applied. 
These children are then evaluated by the fitness functions (Evaluate New Solutions) and added to the offspring pool. These children are also used to assess the performance of the selected LLH (Evaluate Low-Level Heuristic). If the LLH

425 generates good solutions, then it receives a good $r$ reward, or a bad $r$ reward, otherwise. This LLH then has its $t$ set to 0 , and the other LLHs have their $t$ incremented by 1 . If the offspring population is not full yet, then the process described in this paragraph is repeated until all the children are created.

With an offspring population full of solutions, the algorithm can now select the solutions to survive for the next generation (Select Surviving Population) using the replacement strategy implemented by the MOEA. For instance, NSGA-II joins parents and children in a single population, and then ranks them according to their Pareto dominance and their crowding distance [14]. NSGA-II then selects the most well ranked solutions and let them survive for the next generation, whereas the worst ranked solutions are discarded. After this, HITO increments the number of generations (Increment Generation), and continues to the next execution of the loop (if necessary).

At the end of the Loop of Generations step, HITO returns the non-dominated solutions of the current surviving population in the Return Current Population 440 step. The non-dominated solutions returned by HITO are the ones that present the best trade-off between the considered objectives.

The next subsections present, respectively: the LLHs implemented in this work; the measures $r$ and $t$ and how their values are updated for each LLH; and how we adapted the $\mathrm{CF}$ and MAB methods.

\subsection{Low-Level Heuristics}

In this work a LLH is either a crossover operator, or a combination of a mutation operator and a crossover operator. HITO can use any type of operators as LLH, depending just on the problem encoding. In this paper we use three crossover operators and two mutation operators, all for permutation encoding:

450 i) Two Points Crossover (2P), Uniform Crossover and PMX Crossover; and ii) Swap Mutation and Insertion Mutation. 
Table 1 presents the LLHs used in this work, which are composed by the operators mentioned in this subsection. We decided to include LLHs composed only by crossover operators ( $h 1, h 4$ and $h 7)$, because this kind of operator is a distinguishing feature of evolutionary and genetic algorithms [40].

Table 1: Low-level heuristics composition

\begin{tabular}{cccc}
\cline { 2 - 4 } & \multicolumn{3}{c}{ Mutation } \\
\hline Crossover & - & Swap & Insertion \\
\hline Two Points (2P) & $\mathrm{h} 1$ & $\mathrm{~h} 2$ & $\mathrm{~h} 3$ \\
Uniform & $\mathrm{h} 4$ & $\mathrm{~h} 5$ & $\mathrm{~h} 6$ \\
PMX & $\mathrm{h} 7$ & $\mathrm{~h} 8$ & $\mathrm{~h} 9$ \\
\hline
\end{tabular}

\subsection{The measures $r$ and $t$}

As mentioned in the previous sections, $r$ and $t$ are updated in each genetic mating. Therefore, we implemented the credit assignment rules based on this fact. The update rules for $t$ are straightforward: in each mating increment the $t$ value for the LLHs not applied in that mating, and reset the counter to 0 for the applied one. Thus, the $t$ value of a LLH is set in the interval $[0 . .+\infty]$. On the other hand, for each LLH, its $r$ value is computed using the mating parents and the generated children as shown in Equation 6.

$$
r_{h}=\frac{1}{|P| \cdot|C|} \cdot \sum_{p \in P} \sum_{c \in C} \begin{cases}1 & \text { if } c \prec p \\ 0 & \text { if } p \prec c \\ 0.5 & \text { otherwise }\end{cases}
$$

where $r_{h}$ is the computed $r$ value for the applied LLH $h ; P$ is the set of mating parents; $p$ is a parent in $P$; $C$ is the set of children generated in that mating; and $c$ is a child in $C$. One comparison is done for each parent and child and the result of this comparison is summed. If a child dominates a parent $(c \preceq p)$, then 1 is added. If a parent dominates a child ( $p \preceq c$ ), then 0 is added. If both child 
and parent are non-dominated, then 0.5 is added. The sum result is normalized with $1 /(|P| \cdot|C|)$ to encompass algorithms that generate different numbers of children in each mating.

For instance, if two children generated by a LLH $i$ dominate both parents, then $r_{h}=1$. If the two parents dominate both children, then $r_{h}=0$. If both children are non-dominated in relation to their parents, then $r_{h}=0.5$. The higher the value of $r_{h}$, the better the LLH $h$ performed in that mating.

As far as we know, there are no reward measures for credit assignment that use solely the concept of Pareto dominance and only the individuals involved in the mating. In some works, such as $[28,36]$, the reward is computed by comparing the generated solutions with the whole population using quality indicators for each generation or after $n$ (parameter) generations. One of the advantages of our measure is its straightforward implementation using explicitly the well-

475 known Pareto dominance concept. In addition, it is potentially faster to execute, because it uses only the mating solutions and some constant values, instead of complex population based indicators and several solutions. The minor drawback is the positive reward given to a LLH when using the worst possible individuals as parents, as it will usually generate children that dominate their parents.

The next subsections present how these measures are used by CF and MAB.

\subsection{Choice Function Adaptation}

The CF adaptation, proposed in this work, uses the measures $t$ and $r$ directly. However, it still maintains the main features of this CF variation: an exploration and an exploitation factor. The adaptation proposed is a derivation of Equation 2, where the $f_{1}$ function was replaced by the direct value of $r$, and $f_{2}$ was replaced by the direct value of $t$, while the weight parameters $\alpha$ and $\beta$ were kept to balance the exploration and exploitation. Usually the $\alpha$ is set to 1 and $\beta$ to a very low decimal value (0.005 or less), since the $r$ interval is relatively small $([0,1])$ when compared to the $t$ interval $([0 . .+\infty])$. 
To adapt MAB we used the SIMAB [19] method. It is similar to the original equation presented in Subsection 2.2, changing only the reward $r_{i, t}$ and the time factor $t$. In our implementation, the proposed $r$ measure replaces the original instant reward $r_{i, t}$, and the time factor $t$ replaces $\left(t-t_{i}\right)$ in the equations.

In essence, the adapted MAB method is the same as the original [19], because it differs only in the computing of the instant reward and the elapsed time. At the end of the evaluation, a LLH is chosen according to its value regarding Equation 3. The only parameter that needs to be tuned for this method is $C$, which balances the exploration and exploitation factors.

For both methods, $\mathrm{CF}$ and $\mathrm{MAB}$, we used the max strategy selection. If $\mathrm{CF}$ is being used as selection method, then the LLH with the maximum CF value is applied. If MAB is being used, then the LLH with the maximum MAB value is applied. If there is a tie in the $\mathrm{CF} / \mathrm{MAB}$ value between two or more LLHs, then a random tied LLH is selected.

\section{Empirical Evaluation}

The empirical evaluation focuses on the results obtained by HITO when compared to two MOEAs. This section describes the research questions (RQs) that guided this evaluation, used systems, and how the algorithms were configured. Then, it presents the analyses and the results to answer the RQs.

\subsection{Research Questions (RQs)}

RQ1: How is the performance of HITO regarding the ITO problem? This question investigates if HITO can properly solve the considered problem when compared to two other conventional MOEAs: the well-known and widely used NSGA-II, and the state of the art MOEA/DD [34]. In addition, it investigates if HITO can overcome the results achieved by these conventional metaheuristics and by a hyper-heuristic using a random selection. 
Table 2: Details of the systems used in the study

\begin{tabular}{cccccc}
\hline \multirow{2}{*}{ Name } & \multirow{2}{*}{ Paradigm } & \multicolumn{2}{c}{ Units } & & \\
\cline { 3 - 4 } & & Classes & Aspects & Dependencies & LOC \\
MyBatis & OO & 331 & - & 1271 & 23535 \\
AJHSQLDB & AO & 276 & 25 & 1338 & 68550 \\
AJHotDraw & AO & 290 & 31 & 1592 & 18586 \\
BCEL & OO & 45 & - & 289 & 2999 \\
JHotDraw & OO & 197 & - & 809 & 20273 \\
HealthWatcher & AO & 95 & 22 & 399 & 5479 \\
JBoss & OO & 150 & - & 367 & 8434 \\
\hline
\end{tabular}

RQ2: How is the performance of the selection methods CF and MAB? For answering this question, we analyzed the results of both selection methods in order to identify which one provides the best results.

RQ3: Which LLH is the most appropriate to this problem? To answer this question, we analyzed the number of times each LLH (Subsection 5.1) was applied during the execution of HITO.

\subsection{Systems Used in the Study}

The real systems used in this work are detailed in Table 2. The first column presents the name of the systems. The second column presents the paradigm of the system (OO or $\mathrm{AO})$. The third and fourth columns present respectively how many classes (Cls.) and aspects (As.) (both considered units) exist in the system. The fifth column presents the number of dependencies between the units of the system. Finally, the sixth column presents how many Lines of Code 530 (LOC) the system has. Cells with a hyphen (-) represent none.

These are the same systems used in [4]. We chose to generate integration and test orders for these systems for a consistent comparison with this work. Moreover, some of these systems are AO, which inserts a bit of complexity to the problem, due to the increased number of dependencies between the units and to the different possible dependency scenarios. 


\subsection{Experiments Organization}

In order to answer our questions we configured the following algorithms: i) MOEA/DD; ii) NSGA-II; iii) HITO-CF - algorithm that applies HITO with the CF selection method; iv) HITO-MAB - algorithm that applies HITO with the MAB selection method; and v) HITO-R - algorithm that applies HITO with a random selection method. Each algorithm was executed 30 times for each system. The results were compared using the quality indicators hypervolume and Inverted Generational Distance (IGD) [50]. We also computed the KruskalWallis statistical tests (at 95\% significance level) [15] and Vargha-Delaney's Â effect size [2] for the quality indicators' results.

The hypervolume indicator computes the area (or volume when working with more than 2 objectives) of the objective space that a Pareto front dominates [50]. Its calculation is done using a reference point solution, usually the worst of all possible solutions. The advantage of hypervolume is that it takes into account both convergence and diversity. In addition, the software engineer is able to determine if a Pareto front is better by comparing their hypervolume values. The greater the hypervolume value, the better the front is. The IGD indicator, in turn, calculates the distance of the true Pareto front $\left(P F_{\text {true }}\right)$ to another front, thus the lower the IGD of a front, the better this front is.

555 However, for our systems, the PFtrue fronts are unknown. Due to this, we generated an approximated $P F_{\text {true }}$ for each system using the non-dominated solutions of the known Pareto fronts $\left(P F_{\text {known }}\right)$ found by the algorithms. A $P F_{k n o w n}$ front is composed by all the non-dominated solutions found in the 30 executions of an algorithm. Hence, each algorithm found a $P F_{k n o w n}$ for a given system at the end of its 30 executions by joining the 30 resulting fronts. These $P F_{k n o w n}$ fronts were later used to compose the $P F_{\text {true }}$ approximation by joining all non-dominated solutions, and to compute the IGD values for the algorithms.

HITO was implemented using jMetal [17], an object-oriented framework for multi-objective evolutionary optimization. The conventional MOEAs and HITO were executed in all systems and with two sets of objective functions, according to the measures presented in Section 5: i) a set of two objectives (Number of 
Attributes (A) and Number of Operations (O)); and ii) a set of four objectives (A, O, Number of Distinct Return Types (R) and Number of Distinct Parameters $(\mathrm{P})$ ). For all experiments we defined NSGA-II [14] as the MOEA used by HITO, since it is one of the common MOEAs used for solving this problem and within the hyper-heuristics field for comparison [4, 28, 36, 47]. For executing the described algorithms, we used the chromosome representation and the constraints adopted by the approach presented in [4], since this approach was used as a standard approach for the MOEA due to its good results.

The NSGA-II parameters were set according to [4], whereas the MOEA/DD parameters were set according to $[4,34]$. The parameters required by MAB and $\mathrm{CF}$ were set after an empirical tuning. This tuning was done to properly balance the different intervals between the credit assignment metrics $r$ and $t$. The NSGA-II, MOEA/DD, HITO-CF, HITO-MAB and HITO-R parameters for this study are presented in Table 3. The CF weight parameters $(\alpha$ and $\beta)$, and the MAB size of the sliding window $(W)$ and scaling factor $(C)$ are used to balance exploration and exploitation. Furthermore, MOEA/DD has three additional parameters when compared to NSGA-II: a penalty for the Penalty based Boundary Intersection (PBI) decomposition method, a neighborhood size ${ }_{585}(T)$, and a probability to select solutions from the neighborhood $(\delta)$.

Table 3: Parameters

\begin{tabular}{cccc}
\hline Common Parameters & MOEA/DD & HITO-CF & HITO-MAB \\
\hline Population Size: 300 & PBI Penalty: 5.0 & $\alpha: 1.0$ & $W: 150$ \\
Max. Generations: 200 & $T: 20$ & $\beta: 0.00005$ & $C: 5$ \\
& $\delta: 0.9$ & & \\
\hline
\end{tabular}

The mutation and crossover probabilities were set differently. For NSGAII and MOEA/DD, we used a crossover probability of $95 \%$ and a mutation probability of $2 \%$, as described in [4]. For HITO-CF, HITO-MAB and HITO$\mathrm{R}$, we did not use such probabilities because if a LLH is selected by HITO then 
Figure 3: $P F_{k n o w n}$ fronts for MyBatis

Figure 4: $P F_{k n o w n}$ fronts for AJHSQLDB select the operators and configure their probability. This is one of the main advantages of HITO. Moreover, for MOEA/DD with 4 objectives, we had to set the population size to 364 in order to successfully generate weighting vectors.

\subsection{Results}

The obtained results are divided by number of objectives ( 2 and 4 ), but they are discussed together. First, we analyzed the fronts generated by each algorithm. Figures 3-6 depict $P F_{\text {known }}$ fronts for 4 problems with 2 objectives. We presented only the plots that show visible differences between the fronts.

As seen in Figure 3 for MyBatis, NSGA-II, HITO-CF, and HITO-MAB obtained the best fronts with solutions overlapping each other. MOEA/DD was able to find some solutions among the best ones, but some solutions found are dominated by the other algorithms. On the other hand, for AJHSQLDB in Figure 4, MOEA/DD obtained the majority of the non-dominated solutions, and HITO-CF just a few non-dominated solutions. For BCEL (Figure 5), only NSGA-II performed noticeable worse than the other algorithms. Finally, for AJHotDraw depicted in Figure 6, HITO-CF and HITO-MAB obtained all the non-dominated solutions, NSGA-II obtained intermediate solutions, and MOEA/DD and HITO-R obtained overlapping fronts with the worst solutions.

Because the plots cannot reveal the best algorithm, the usage of quality indicators is necessary. Tables 4 and 5 show the average hypervolume found by the algorithms for 2 and 4 objectives respectively. The average was computed using the 30 fronts found by each algorithm and for each system. For both tables,

Figure 5: $P F_{\text {known }}$ fronts for BCEL 
Figure 6: $P F_{\text {known }}$ fronts for AJHotDraw

the first column shows the system, and the second, third, fourth, fifth and sixth columns show respectively the results obtained by MOEA/DD, NSGA-II, HITOCF, HITO-MAB and HITO-R. Values highlighted in bold are the best values or the values statistically equivalent to the best one using the Kruskal-Wallis test with $95 \%$ of significance. It is important to note that the Kruskal-Wallis test was performed using the 30 hypervolume values (one value for each independent run) of the resulting fronts of each algorithm, and not the average values alone.

Table 4: Hypervolume averages found for 2 objectives

\begin{tabular}{|c|c|c|c|c|c|}
\hline System & MOEA/DD & NSGA-II & HITO-CF & НITO-МАВ & HITO-R \\
\hline MyBatis & $\begin{array}{c}6,81 \mathrm{E}-1 \\
(4,41 \mathrm{E}-2)\end{array}$ & $\begin{array}{c}7,65 \mathrm{E}-1 \\
(3,96 \mathrm{E}-2)\end{array}$ & $\begin{array}{c}8,00 \mathrm{E}-1 \\
(2,71 \mathrm{E}-2)\end{array}$ & $\begin{array}{c}7,83 \mathrm{E}-1 \\
(3,06 \mathrm{E}-2)\end{array}$ & $\begin{array}{c}6,88 \mathrm{E}-1 \\
(2,37 \mathrm{E}-2)\end{array}$ \\
\hline AJHSQLDB & $\begin{array}{c}7,41 \mathrm{E}-1 \\
(7,94 \mathrm{E}-2)\end{array}$ & $\begin{array}{c}5,86 \mathrm{E}-1 \\
(1,12 \mathrm{E}-1)\end{array}$ & $\begin{array}{c}6,90 \mathrm{E}-1 \\
(6,76 \mathrm{E}-2)\end{array}$ & $\begin{array}{c}6,45 \mathrm{E}-1 \\
(1,06 \mathrm{E}-1)\end{array}$ & $\begin{array}{c}4,11 \mathrm{E}-1 \\
(6,86 \mathrm{E}-2)\end{array}$ \\
\hline AJHotDraw & $\begin{array}{c}5,74 \mathrm{E}-1 \\
(7,95 \mathrm{E}-2)\end{array}$ & $\begin{array}{c}5,91 \mathrm{E}-1 \\
(1,15 \mathrm{E}-1)\end{array}$ & $\begin{array}{c}8,16 \mathrm{E}-1 \\
(8,56 \mathrm{E}-2)\end{array}$ & $\begin{array}{c}8,11 \mathrm{E}-1 \\
(9,30 \mathrm{E}-2)\end{array}$ & $\begin{array}{c}5,52 \mathrm{E}-1 \\
(9,27 \mathrm{E}-2)\end{array}$ \\
\hline BCEL & $\begin{array}{c}8,08 \mathrm{E}-1 \\
(1,25 \mathrm{E}-2)\end{array}$ & $\begin{array}{c}2,87 \mathrm{E}-1 \\
(5,15 \mathrm{E}-3)\end{array}$ & $\begin{array}{c}7,87 \mathrm{E}-1 \\
(8,01 \mathrm{E}-3)\end{array}$ & $\begin{array}{c}7,81 \mathrm{E}-1 \\
(3,51 \mathrm{E}-2)\end{array}$ & $\begin{array}{c}6,95 \mathrm{E}-1 \\
(5,44 \mathrm{E}-2)\end{array}$ \\
\hline JHotDraw & $\begin{array}{c}8,06 \mathrm{E}-1 \\
(1,13 \mathrm{E}-1)\end{array}$ & $\begin{array}{c}4,72 \mathrm{E}-1 \\
(2,67 \mathrm{E}-1)\end{array}$ & $\begin{array}{c}8,53 \mathrm{E}-1 \\
(1,26 \mathrm{E}-1)\end{array}$ & $\begin{array}{c}9,19 \mathrm{E}-1 \\
(7,53 \mathrm{E}-2)\end{array}$ & $\begin{array}{c}7,88 \mathrm{E}-1 \\
(1,25 \mathrm{E}-1)\end{array}$ \\
\hline HealthWatcher & $\begin{array}{c}8,17 \mathrm{E}-1 \\
(5,45 \mathrm{E}-2)\end{array}$ & $\begin{array}{c}8,52 \mathrm{E}-1 \\
(2,61 \mathrm{E}-2)\end{array}$ & $\begin{array}{c}8,65 \mathrm{E}-1 \\
(2,57 \mathrm{E}-2)\end{array}$ & $\begin{array}{c}8,80 \mathrm{E}-1 \\
(4,36 \mathrm{E}-2)\end{array}$ & $\begin{array}{c}8,78 \mathrm{E}-1 \\
(4,14 \mathrm{E}-2)\end{array}$ \\
\hline JBoss & $\begin{array}{c}9,50 \mathrm{E}-1 \\
(1,40 \mathrm{E}-1)\end{array}$ & $\begin{array}{l}5,38 \mathrm{E}-3 \\
(0,00 \mathrm{E} 0)\end{array}$ & $\begin{array}{c}8,81 \mathrm{E}-1 \\
(1,85 \mathrm{E}-1)\end{array}$ & $\begin{array}{c}9,32 \mathrm{E}-1 \\
(1,32 \mathrm{E}-1)\end{array}$ & $\begin{array}{c}7,16 \mathrm{E}-1 \\
(2,45 \mathrm{E}-1)\end{array}$ \\
\hline
\end{tabular}

Because we normalized the objective values of all solutions into the interval of $[0 . .1]$, the worst possible value for the objectives is 1 (given that the problem is a minimization one). Hence, adding a small offset of 0.01 , the reference point for the hypervolume calculation was set to $(1.01,1.01)$ for 2 objectives and $(1.01,1.01,1.01,1.01)$ for 4 objectives.

For every system, on both objective sets, HITO was able to outperform NSGA-II by finding a greater hypervolume average. Only in four cases (HealthWatcher and MyBatis with 2 objectives, and MyBatis and AJHSQLDB with 4 
Table 5: Hypervolume averages found for 4 objectives

\begin{tabular}{cccccc}
\hline System & MOEA/DD & NSGA-II & HITO-CF & HITO-MAB & HITO-R \\
\hline \multirow{2}{*}{ MyBatis } & $1,09 \mathrm{E}-1$ & $\mathbf{7 , 1 3 E - 1}$ & $\mathbf{7 , 3 0 E - 1}$ & $6,58 \mathrm{E}-1$ & $5,44 \mathrm{E}-1$ \\
& $(3,25 \mathrm{E}-2)$ & $\mathbf{( 4 , 9 9 E - 2 )}$ & $\mathbf{( 3 , 7 3 E - 2 )}$ & $(4,76 \mathrm{E}-2)$ & $(4,20 \mathrm{E}-2)$ \\
AJHSQLDB & $6,69 \mathrm{E}-2$ & $\mathbf{7 , 2 6 E - 1}$ & $\mathbf{7 , 8 0 E - 1}$ & $\mathbf{7 , 1 1 E}-\mathbf{1}$ & $4,90 \mathrm{E}-1$ \\
& $(3,20 \mathrm{E}-2)$ & $\mathbf{( 1 , 0 1 E - 1 )}$ & $\mathbf{( 7 , 6 5 E - 2 )}$ & $\mathbf{( 9 , 5 9 E - 2 )}$ & $(5,31 \mathrm{E}-2)$ \\
AJHotDraw & $4,99 \mathrm{E}-1$ & $6,94 \mathrm{E}-1$ & $\mathbf{7 , 9 9 E - 1}$ & $\mathbf{7 , 9 1 E - 1}$ & $5,68 \mathrm{E}-1$ \\
& $(4,86 \mathrm{E}-2)$ & $(5,47 \mathrm{E}-2)$ & $\mathbf{( 6 , 1 1 E - 2 )}$ & $\mathbf{( 6 , 0 9 E - 2 )}$ & $(5,58 \mathrm{E}-2)$ \\
BCEL & $6,55 \mathrm{E}-1$ & $8,06 \mathrm{E}-2$ & $\mathbf{7 , 3 1 E - 1}$ & $\mathbf{7 , 2 0 E - 1}$ & $6,10 \mathrm{E}-1$ \\
& $(3,06 \mathrm{E}-2)$ & $(9,85 \mathrm{E}-4)$ & $\mathbf{( 8 , 9 9 E - 3 )}$ & $\mathbf{( 7 , 2 0 E - 3 )}$ & $(2,93 \mathrm{E}-2)$ \\
JHotDraw & $8,53 \mathrm{E}-1$ & $8,28 \mathrm{E}-1$ & $\mathbf{9 , 5 1 E - 1}$ & $\mathbf{9 , 4 6 E - 1}$ & $8,60 \mathrm{E}-1$ \\
& $(9,25 \mathrm{E}-2)$ & $(1,30 \mathrm{E}-1)$ & $\mathbf{( 4 , 1 5 E - 2 )}$ & $\mathbf{( 5 , 4 9 E - 2 )}$ & $(6,67 \mathrm{E}-2)$ \\
HealthWatcher & $\mathbf{7 , 3 1 E - 1}$ & $5,69 \mathrm{E}-1$ & $\mathbf{6 , 4 1 E - 1}$ & $6,10 \mathrm{E}-1$ & $\mathbf{6 , 4 4 E - 1}$ \\
& $\mathbf{( 1 , 7 1 E - 1 )}$ & $(4,39 \mathrm{E}-2)$ & $\mathbf{( 1 , 3 6 E - 1 )}$ & $(8,49 \mathrm{E}-2)$ & $(\mathbf{1}, \mathbf{2 9 E}-\mathbf{1})$ \\
JBoss & $\mathbf{9 , 8 3 E - 1}$ & $3,24 \mathrm{E}-3$ & $\mathbf{9 , 7 0 E - 1}$ & $\mathbf{9 , 9 4 E - 1}$ & $7,67 \mathrm{E}-1$ \\
& $(\mathbf{9 , 4 9 E - 2 )}$ & $(0,00 \mathrm{E} 0)$ & $\mathbf{( 9 , 5 7 E - 2 )}$ & $\mathbf{( 3 , 3 7 E - 2 )}$ & $(2,17 \mathrm{E}-1)$ \\
\hline
\end{tabular}

objectives) NSGA-II yielded statistically equivalent results to the ones of HITO$\mathrm{CF}$ and HITO-MAB. For MyBatis with 4 objectives, NSGA-II was statistically better than HITO-MAB, but yet HITO-CF outperformed it. MOEA/DD could statistically outperform all the other algorithms only in BCEL with 2 objectives. Apart from that, it performed equally or worse than HITO-CF and/or HITOMAB, sometimes even losing to NSGA-II. We expected a better performance from this state of the art algorithm, specially for 4 objectives where the benefits of decomposition could be better availed.

We also computed the IGD values for all 30 fronts obtained by the algorithms in each system. The reference front was generated joining all non-dominated solutions found in this experiment. These results are presented in Tables 6 and 7 .

MOEA/DD was only able to statistically outperform the other algorithms in BCEL with 2 objectives. Besides that, MOEA/DD presented mixed results when compared to HITO-CF and HITO-MAB, always showing worse or equal results. For the biggest two systems with 4 objectives, NSGA-II obtained statistically equivalent results to HITO-CF. HITO-CF on the other hand presented the best overall results considering IGD. Except for BCEL with 2 objectives, 
Table 6: IGD averages found for 2 objectives

\begin{tabular}{|c|c|c|c|c|c|}
\hline System & MOEA/DD & NSGA-II & HITO-CF & НІТО-МАВ & HITO-R \\
\hline \multirow[t]{2}{*}{ MyBatis } & $2,95 \mathrm{E}-2$ & $1,41 \mathrm{E}-2$ & $8,93 \mathrm{E}-3$ & 1,12E-2 & $2,60 \mathrm{E}-2$ \\
\hline & $(7,89 \mathrm{E}-3)$ & $(6,63 \mathrm{E}-3)$ & $(4,00 \mathrm{E}-3)$ & $(4,74 \mathrm{E}-3)$ & $(3,88 \mathrm{E}-3)$ \\
\hline \multirow[t]{2}{*}{ AJHSQLDB } & $5,50 \mathrm{E}-2$ & $1,12 \mathrm{E}-1$ & $8,03 \mathrm{E}-2$ & $9,52 \mathrm{E}-2$ & $1,88 \mathrm{E}-1$ \\
\hline & $(2,18 \mathrm{E}-2)$ & $(3,72 \mathrm{E}-2)$ & $(2,10 \mathrm{E}-2)$ & $(3,53 \mathrm{E}-2)$ & $(2,78 \mathrm{E}-2)$ \\
\hline \multirow[t]{2}{*}{ AJHotDraw } & $6,10 \mathrm{E}-1$ & $5,02 \mathrm{E}-1$ & $2,11 \mathrm{E}-1$ & $2,11 \mathrm{E}-1$ & $5,22 \mathrm{E}-1$ \\
\hline & $(1,54 \mathrm{E}-1)$ & $(2,11 \mathrm{E}-1)$ & $(1,20 \mathrm{E}-1)$ & $(1,37 \mathrm{E}-1)$ & $(1,60 \mathrm{E}-1)$ \\
\hline \multirow[t]{2}{*}{ BCEL } & $7,04 \mathrm{E}-3$ & $1,26 \mathrm{E}-1$ & $1,26 \mathrm{E}-2$ & $1,41 \mathrm{E}-2$ & $3,05 \mathrm{E}-2$ \\
\hline & $(3,50 \mathrm{E}-3)$ & $(1,39 \mathrm{E}-3)$ & $(2,07 \mathrm{E}-3)$ & $(6,52 \mathrm{E}-3)$ & $(9,84 \mathrm{E}-3)$ \\
\hline \multirow[t]{2}{*}{ JHotDraw } & $1,77 \mathrm{E} 0$ & $5,90 \mathrm{E} 0$ & 1,40E0 & $6,55 \mathrm{E}-1$ & 1,27E0 \\
\hline & $(1,29 \mathrm{E} 0)$ & $(5,16 \mathrm{E} 0)$ & $(1,34 \mathrm{E} 0)$ & $(7,74 \mathrm{E}-1)$ & $(1,28 \mathrm{E} 0)$ \\
\hline \multirow[t]{2}{*}{ HealthWatcher } & 0,00E0 & 0,00E0 & 0,00E0 & 0,00E0 & 0,00E0 \\
\hline & $(0,00 \mathrm{E} 0)$ & $(0,00 \mathrm{E} 0)$ & $(0,00 \mathrm{E} 0)$ & $(0,00 \mathrm{E} 0)$ & $(0,00 \mathrm{E} 0)$ \\
\hline \multirow[t]{2}{*}{ JBoss } & 0,00E0 & 0,00E0 & 0,00E0 & 0,00E0 & 0,00E0 \\
\hline & $(0,00 \mathrm{E} 0)$ & $(0,00 \mathrm{E} 0)$ & $(0,00 \mathrm{E} 0)$ & $(0,00 \mathrm{E} 0)$ & $(0,00 \mathrm{E} 0)$ \\
\hline
\end{tabular}

Table 7: IGD averages found for 4 objectives

\begin{tabular}{cccccc}
\hline System & MOEA/DD & NSGA-II & HITO-CF & HITO-MAB & HITO-R \\
\hline \multirow{2}{*}{ MyBatis } & $4,89 \mathrm{E}-2$ & $\mathbf{5 , 5 9 E - 3}$ & $\mathbf{5 , 1 2 E - 3}$ & $7,58 \mathrm{E}-3$ & $1,17 \mathrm{E}-2$ \\
& $(6,38 \mathrm{E}-3)$ & $\mathbf{( 1 , 2 2 E - 3 )}$ & $\mathbf{( 1 , 1 1 E - 3 )}$ & $(1,77 \mathrm{E}-3)$ & $(1,95 \mathrm{E}-3)$ \\
AJHSQLDB & $1,72 \mathrm{E}-1$ & $\mathbf{2 , 1 1 E - 2}$ & $\mathbf{1 , 6 6 E - 2}$ & $\mathbf{2 , 4 0 E - 2}$ & $5,32 \mathrm{E}-2$ \\
& $(2,13 \mathrm{E}-2)$ & $\mathbf{( 1 , 0 7 E - 2 )}$ & $\mathbf{( 7 , 2 0 E - 3 )}$ & $\mathbf{( 1 , 0 8 E - 2 )}$ & $(9,67 \mathrm{E}-3)$ \\
AJHotDraw & $8,99 \mathrm{E}-2$ & $4,48 \mathrm{E}-2$ & $\mathbf{3 , 2 2 E - 2}$ & $\mathbf{3 , 2 7 E - 2}$ & $8,49 \mathrm{E}-2$ \\
& $(1,82 \mathrm{E}-2)$ & $(8,90 \mathrm{E}-3)$ & $\mathbf{( 8 , 9 0 E - 3 )}$ & $\mathbf{( 8 , 8 9 E - 3 )}$ & $(1,89 \mathrm{E}-2)$ \\
BCEL & $1,90 \mathrm{E}-2$ & $1,28 \mathrm{E}-1$ & $\mathbf{9 , 6 3 E - 3}$ & $\mathbf{9 , 0 3 E - 3}$ & $2,11 \mathrm{E}-2$ \\
& $(2,79 \mathrm{E}-3)$ & $(1,80 \mathrm{E}-4)$ & $\mathbf{( 2 , 6 0 E - 3 )}$ & $\mathbf{( 9 , 1 5 E - 4 )}$ & $(2,52 \mathrm{E}-3)$ \\
JHotDraw & $2,36 \mathrm{E}-1$ & $2,05 \mathrm{E}-1$ & $\mathbf{9 , 9 1 E - 2}$ & $\mathbf{9 , 5 0 E - 2}$ & $1,74 \mathrm{E}-1$ \\
& $(1,14 \mathrm{E}-1)$ & $(1,35 \mathrm{E}-1)$ & $\mathbf{( 3 , 7 9 E - 2 )}$ & $\mathbf{( 4 , 3 1 E - 2 )}$ & $(5,48 \mathrm{E}-2)$ \\
HealthWatcher & $\mathbf{0 , 0 0 E 0}$ & $\mathbf{0 , 0 0 E 0}$ & $\mathbf{0 , 0 0 E 0}$ & $\mathbf{0 , 0 0 E 0}$ & $\mathbf{0 , 0 0 E 0}$ \\
& $(\mathbf{0 , 0 0 E 0})$ & $(\mathbf{0 , 0 0 E 0})$ & $\mathbf{( 0 , 0 0 E 0 )}$ & $\mathbf{( 0 , 0 0 E 0 )}$ & $(\mathbf{0 , 0 0 E 0})$ \\
\multirow{2}{*}{ JBoss } & $\mathbf{0 , 0 0 E 0}$ & $\mathbf{0 , 0 0 E 0}$ & $\mathbf{0 , 0 0 E 0}$ & $\mathbf{0 , 0 0 E 0}$ & $\mathbf{0 , 0 0 E 0}$ \\
& $(\mathbf{0 , 0 0 E 0 )}$ & $\mathbf{( 0 , 0 0 E 0 )}$ & $\mathbf{( 0 , 0 0 E 0 )}$ & $\mathbf{( 0 , 0 0 E 0 )}$ & $(\mathbf{0 , 0 0 E 0})$ \\
\hline
\end{tabular}

HITO-CF was always better or equal to the other algorithms. For both quality indicators, HITO-R performed statistically worse or equal to HITO.

The HealthWatcher and JBoss instances appear to be the easiest to solve, since almost all algorithms yielded statistically equivalent results. Furthermore, when analyzing IGD, we observed that all algorithms were able to find the same 
650 are in fact two of the smallest system used in this study, hence the similarity of the results was expected.

We also calculated the Vargha-Delaney's Â effect size [2]. While KruskalWallis calculates if there is statistical difference between groups of data, the $A / B$ of data. According to [2], the $\hat{A}$ value varies between $[0,1]$, where 0.5 represents absolute no difference between the two groups, values below 0.5 represent that group $A$ loses to group $B$, and values above 0.5 represent that $B$ loses to group $A$. Values in ]0.44,0.56[ represent negligible differences, values in $660[0.56,0.64[$ and $] 0.36,0.44]$ represent small differences, values in $[0.64,0.71[$ and ]0.29, 0.44] represent medium differences, and values in $[0.0,0.29]$ and $[0.71,1.0]$ represent large differences. A negligible magnitude usually does not yield statistical difference. The small and medium magnitudes represent small and medium differences between the values, and may or not yield statistical differences. Finally, a large magnitude represents a significantly large difference that usually can be seen in the numbers without a lot of effort.

Because we have computed the $\hat{A}$ value for each group comparison, in all systems, for both sets of objectives, and for both hypervolume and IGD values, we present the results in the format of boxplots with HITO-CF as group $A$. These boxplots are presented in Figures 7 to 10 . The lower the values, the worse the results of HITO-CF in comparison to the respective algorithm.

Figure 7: Hypervolume effect size for 2 objectives

Figure 8: Hypervolume effect size for 4 objectives

Figure 9: IGD effect size for 2 objectives

The effect size results endorse what we observed on the hypervolume and 
Figure 10: IGD effect size for 4 objectives

Table 8: Average number of low-level heuristic applications by HITO-CF with 2 objectives

\begin{tabular}{c|c|c|c|c|c|c|c}
\hline LLH & MyBatis & AJHSQLDB & AJHotDraw & BCEL & JHotDraw & HealthWatcher & JBoss \\
\hline $\mathbf{2 P}$ & $\mathbf{1 3 2 3 2}$ & $\mathbf{1 3 3 6 7}$ & $\mathbf{1 1 6 6 1}$ & 1117 & $\mathbf{9 1 2 1}$ & $\mathbf{1 0 7 9 8}$ & 4690 \\
2P+Swap & 4004 & 3758 & 3024 & 843 & 2894 & 3124 & 2991 \\
2P+Insertion & 5856 & 5879 & 4437 & 950 & 4082 & 4317 & 3473 \\
\hline Uniform & 1218 & 843 & 1093 & 808 & 1468 & 1932 & 2282 \\
Uniform+Swap & 1010 & 736 & 1055 & 729 & 1392 & 1675 & 2242 \\
Uniform+Insertion & 1092 & 755 & 1077 & 764 & 1424 & 1743 & 2264 \\
\hline PMX & 998 & 2376 & 4378 & $\mathbf{2 3 0 9 8}$ & 5928 & 2559 & $\mathbf{7 0 2 9}$ \\
PMX+Swap & 1115 & 970 & 1459 & 750 & 1672 & 1760 & 2402 \\
PMX+Insertion & 1320 & 1163 & 1664 & 786 & 1863 & 1938 & 2474 \\
\hline
\end{tabular}

IGD comparisons. HITO-CF performed very similar to HITO-MAB in almost all cases. HITO-CF usually performed better than HITO-R and NSGA-II with large differences. Lastly, when comparing HITO-CF with MOEA/DD, we observed more balanced results. Comparing the results for 2 objectives, HITO-CF showed effect size medians inside the small difference ranges, favorable in the hypervolume comparisons and unfavorable in the IGD comparison. However, for 4 objectives, MOEA/DD obtained worse results than HITO-CF, for both hypervolume and IGD, with medians in the large difference range.

We collected the average number of times each LLH was applied during the optimization. These averages were calculated using the 30 executions of each algorithm and are shown in Tables 8, 9, 10 and 11. The first two tables show the averages of HITO-CF and HITO-MAB with 2 objectives, and the last two the averages with 4 objectives. The greatest values are highlighted in bold.

We observed in all cases that the most applied LLHs are the ones composed only by crossover operators $(h 1-2 \mathrm{P}$ Crossover, $h 4$ - Uniform Crossover and h7 - PMX Crossover). Moreover, these three LLHs were applied more often than their respective LLHs with mutation operators. The most applied LLH is $h 1$ (2P Crossover), followed by $h 7$ (PMX Crossover). In every scenario, $h 7$ has a greater number of applications for BCEL and JBoss. Additionally, $h 7$ was applied more often in JHotDraw by HITO-MAB with 2 objectives. Apart from 
Table 9: Average number of low-level heuristic applications by HITO-MAB with 2 objectives

\begin{tabular}{c|c|c|c|c|c|c|c|c}
\hline System & \multicolumn{2}{|c|}{ MyBatis } & AJHSQLDB & AJHotDraw & BCEL & JHotDraw & HealthWatcher & JBoss \\
\hline 2P & $\mathbf{8 8 6 8}$ & $\mathbf{9 9 5 4}$ & $\mathbf{9 5 9 3}$ & 2882 & 7366 & $\mathbf{6 2 5 4}$ & 3318 \\
2P+Swap & 4456 & 4264 & 2900 & 1571 & 2713 & 4129 & 1887 \\
2P+Insertion & 5954 & 6180 & 4297 & 1956 & 4373 & 5435 & 2582 \\
\hline Uniform & 1419 & 1020 & 1209 & 1445 & 1266 & 1697 & 1163 \\
Uniform+Swap & 1219 & 1026 & 1166 & 1424 & 1288 & 1515 & 1244 \\
Uniform+Insertion & 1289 & 991 & 1223 & 1508 & 1402 & 1542 & 1206 \\
\hline PMX & 2111 & 2837 & 5632 & $\mathbf{1 5 4 1 8}$ & $\mathbf{7 4 1 4}$ & 4241 & $\mathbf{1 4 7 7 9}$ \\
PMX+Swap & 2057 & 1509 & 1623 & 1537 & 1653 & 2154 & 1509 \\
PMX+Insertion & 2473 & 2063 & 2204 & 2105 & 2372 & 2878 & 2159 \\
\hline
\end{tabular}

Table 10: Average number of low-level heuristic applications by HITO-CF with 4 objectives

\begin{tabular}{c|c|c|c|c|c|c|c|c}
\hline System & MyBatis & AJHSQLDB & AJHotDraw & BCEL & JHotDraw & HealthWatcher & JBoss \\
\hline 2P & $\mathbf{1 4 4 3 0}$ & $\mathbf{1 4 3 3 6}$ & $\mathbf{1 4 7 9 6}$ & 389 & $\mathbf{1 3 1 7 1}$ & $\mathbf{1 0 2 4 1}$ & 4555 \\
2P+Swap & 4398 & 4096 & 3505 & 348 & 3044 & 3175 & 2991 \\
2P+Insertion & 6580 & 6198 & 6190 & 353 & 4861 & 4406 & 3503 \\
\hline Uniform & 981 & 974 & 440 & 334 & 875 & 2178 & 2139 \\
Uniform+Swap & 706 & 786 & 390 & 313 & 768 & 1732 & 2095 \\
Uniform+Insertion & 933 & 887 & 419 & 316 & 819 & 1862 & 2116 \\
\hline PMX & 499 & 1340 & 2346 & $\mathbf{2 7 1 6 6}$ & 3701 & 2649 & $\mathbf{7 8 2 1}$ \\
PMX+Swap & 619 & 579 & 809 & 308 & 1172 & 1733 & 2279 \\
PMX+Insertion & 699 & 650 & 952 & 318 & 1434 & 1869 & 2347 \\
\hline
\end{tabular}

Table 11: Average number of low-level heuristic applications by HITO-MAB with 4 objectives

\begin{tabular}{|c|c|c|c|c|c|c|c|}
\hline System & MyBatis & AJHSQLDB & AJHotDraw & $\mid$ BCEL $\mid$ & |JHotDraw $\mid$ & $\mid$ HealthWatcher $\mid$ & JBoss \\
\hline $2 P$ & 8628 & 9411 & 10362 & 2116 & 8343 & 6551 & 3794 \\
\hline $2 P+S w a p$ & 4298 & 4455 & 3458 & 1895 & 3477 & 4129 & 2158 \\
\hline $2 \mathrm{P}+$ Insertion & 5650 & 6658 & 5708 & 1948 & 5125 & 5121 & 2731 \\
\hline Uniform & 1466 & 1155 & 1266 & 1557 & 1328 & 1669 & 1308 \\
\hline Uniform +Swap & 1393 & 1130 & 1146 & 1638 & 1312 & 1450 & 1343 \\
\hline Uniform + Insertion & 1456 & 1175 & 1198 & 1617 & 1384 & 1506 & 1341 \\
\hline PMX & 2233 & 2349 & 2672 & 15322 & 4669 & 4504 & 13436 \\
\hline PMX+Swap & 2147 & 1582 & 1535 & 1713 & 1747 & 2148 & 1572 \\
\hline PMX+Insertion & 2576 & 1930 & 2501 & 2040 & 2460 & 2768 & 2163 \\
\hline
\end{tabular}


these situations, $h 1$ was always the most applied LLH.

The number of applications of each LLH is correlated to its overall performance during the optimization. If a given LLH was applied more than others, then it means that this LLH constantly held a greater score throughout the process, and hence generated better children than others. Therefore, these numbers point out that, regardless of which selection method is being used, the PMX Crossover operator is the most suitable for BCEL and JHotDraw, and the Two Points Crossover operator is usually the most suitable for the remaining systems. Furthermore, we observed that in most of the cases, the LLHs composed by Simple Insertion Mutation were applied more often than the LLHs composed by the same crossover and Swap Mutation. It shows that Simple Insertion Mutation is overall the best mutation operator for this problem.

Using only crossover during the optimization generates the best children in this kind of problem, whereas the usage of mutation operators can provide worse solutions in some cases. The reason behind this may be that, changing a lot the sequence of the genes is not always beneficial for permutation problems such as this one. This can be evidenced by the functionality of the operators. The crossover operators strictly use the sequence of the parents to generate the children, whereas the mutation operators rely on randomness to insert diversity. Going even further, Simple Insertion Mutation is the best mutation operator because it changes the position of only one random gene, in contrast to Swap Mutation that changes the position of two random genes. In parallel, Two Points genes provided by the parents, whereas Uniform Crossover (the crossover least often applied) is the worst one because it performs the most drastic changes.

However, even though randomness is not always beneficial, the mutation operators should not simply be discarded, since they can provide diversity for the population in some moments of the search process. If the mutation operators were useless, then the selection methods would have selected less often such LLHs. In some cases, the LLHs with mutation operators were applied more than the ones without these operators, which is understandable given the role that 
the mutation plays in the evolution. Further investigations on the functionality of these operators in the hyper-heuristic field must be done in order to provide a more comprehensive insight about their impact on the optimization process. Nevertheless, the real advantage here is that HITO can identify when it is preferable to apply each kind of operator and then can perform accordingly.

\subsection{Answering the Research Questions}

RQ1: How is the performance of HITO regarding the ITO problem?

As seen in the previous subsection, HITO (CF or MAB) was able to outperform or equal NSGA-II and HITO-R in all systems and for both sets of objectives regarding the hypervolume and IGD indicators. When compared to MOEA/DD, in general, HITO was statistically better or equal, but MOEA/DD was the only algorithm able to outperform HITO with statistical difference in one system. The effect size also supported these facts, since HITO usually outperformed NSGA-II, MOEA/DD and HITO-R with large or medium differences. Furthermore, the IGD values show that HITO can also find the best $P F_{\text {known }}$ fronts for most systems, except BCEL where MOEA/DD stood out as the best algorithm. Taking these results into account, it is possible to assert that HITO can properly solve this problem, and usually with better performance than an approach using only a conventional or state of the art MOEA. These positive results emphasize even more the applicability of hyper-heuristics in SBSE.

RQ2: How is the performance of CF and MAB?

${ }_{745}$ Even though HITO-CF performed slightly better than HITO-MAB, it is not possible to determine for sure if it is superior. The results of the Kruskal-Wallis test for the algorithms using both selection methods rarely showed statistical differences, and the effect size values usually presented negligible, small or medium magnitudes. We expected MAB to yield significant better results, since some of its equations focus on quickly identifying changes in the ranking of LLHs [19]. For future works, we intend to tweak the rewarding credit assignment equation (proposed in Subsection 5.2) for a better compatibility with MAB.

RQ3: Which LLH is the most appropriate for this problem? 
Overall, the best LLHs are composed only by crossover operators, specifically $h 1$ (Two Points Crossover) and $h 7$ (PMX Crossover). The PMX operator is the most suitable crossover for BCEL and JHotDraw, whereas Two Points Crossover is the best one for the other systems. Regarding the mutation operators, Simple Insertion Mutation performed better due to its small changes in the genes order.

\subsection{Threats to Validity}

Some systems used in the experimentation are small when compared to the others, such as JBoss, HealthWatcher and JHotDraw. For instance, almost in all cases the results found by the algorithms for HealthWatcher are very similar, therefore, the Kruskal-Wallis test showed statistical equivalence between the results. The IGD indicator also showed that all algorithms found the same nondominated solutions for HealthWatcher and JBoss. Thus, the results for these systems cannot be generalized for larger systems.

The rewarding measure and the $\mathrm{CF}$ and $\mathrm{MAB}$ adaptations used by HITO may be accurate only for HITO using NSGA-II. For instance, if SPEA2 is used by HITO, then the time factor $t$ of each LLH will scale quicker and the performance of HITO may be affected, since SPEA2 generates only one child per mating in its implementation of the framework jMetal.

We used a fixed set of operators. Different operators can lead to different results. Therefore, we intend to evaluate other sets of operators in future work, such as newer operators, bigger sets of operators, different combinations of operators (e.g. no crossover, multiple mutation), and so on.

The MOEAs compared in this study were previously proposed and tested in continuous problems. This might be one explanation for their inferiority in comparison to HITO in the experimentation shown in this paper. Nevertheless, if the user is planning to arbitrarily choose and configure a MOEA for this problem, he/she should consider a hyper-heuristic instead, since it might be a better choice for this kind of problem. 


\section{Concluding Remarks}

In this study we introduced HITO, a hyper-heuristic for the ITO problem. HITO dynamically selects LLHs (combination of crossover and mutation opperformance of each LLH. This metric is straightforward and uses only the parents and children involved in the mating. The reward is then used by selection methods, such as Choice Function ( $\mathrm{CF}$ ) and Multi-Armed Bandit (MAB), to select the best LLH at each mating. HITO was designed to encompass a set of steps and to use inputs in order to allow the tester to flexibly personalize its execution. Therefore, the tester can provide his/her LLHs, MOEAs, software metrics to be used as fitness functions, selection methods and problem instances, according to his/her goals. However, the tester does not need to select the operators, nor to configure the algorithm parameters, because HITO makes such decisions. This is an advantage of using hyper-heuristics.

HITO also presents other advantages. Even though we only used HITO to the ITO problem, it is a generic hyper-heuristic that can be applied for other software engineering problems and for any MOEA. For example, HITO can be used with SPEA2 [49] for solving problems such as prioritization and selection of test cases, considering different objectives, such as coverage, size of test set, memory consumption, etc.

To evaluate HITO performance, we designed experiments with 2 and 4 objectives and used 7 real systems in an empirical evaluation, in both object-oriented and aspect-oriented contexts. We implemented the $\mathrm{CF}$ and MAB methods and compared HITO with its random version, with a well-known MOEA (NSGAII [14]), and with a state of the art MOEA (MOEA/DD [34]). HITO was able to outperform or statistically match NSGA-II and the random hyper-heuristic for all systems and sets of objectives. When compared to MOEA/DD, the results were also favorable in overall, but MOEA/DD was able to outperform HITO in one instance of the problem. The effect size test showed that $\mathrm{CF}$ and MAB 
performed similarly, but CF was slightly better. By analyzing the number of applications of each LLH, we observed that some crossover and mutation operators are more suitable for the systems used, specifically Two Points Crossover and Simple Insertion Mutation.

Future works should to test the flexibility of HITO using other MOEAs and other problems to address its performance across the SBSE field. We also intend to increase the accuracy of the proposed rewarding measure by allowing a fine-grained evaluation of LLHs that hopefully will improve the results. Furthermore, HITO shall be evaluated using other selection methods and other search operator. Moreover, other hyper-heuristic techniques can be used, such as on-line parameter tuning, metaheuristic selection and acceptance functions.

Instead of working solely on the search space of operators, hyper-heuristics can be employed to identify key characteristics of each system module. Later, these characteristics can be used to better guide the units ordering. Finally, we will investigate why mutation operators were not so useful for this problem.

\section{Acknowledgment}

This work is supported by the Brazilian funding agencies CAPES and CNPq under the grants: 307762/2015-7 and 473899/2013-2.

\section{References}

[1] Ahandani, M. A., Baghmisheh, M. T. V., Zadeh, M. A. B., Ghaemi, S., 2012. Hybrid particle swarm optimization transplanted into a hyperheuristic structure for solving examination timetabling problem. Swarm and Evolutionary Computation 7 (0), 21-34.

835

[2] András Vargha, H. D. D., 2000. A Critique and Improvement of the "CL" Common Language Effect Size Statistics of McGraw and Wong. Journal of Educational and Behavioral Statistics 25 (2), 101-132. 
[3] Assunção, W. K. G., Colanzi, T. E., Pozo, A., Vergilio, S. R., 2011. Establishing integration test orders of classes with several coupling measures. In: Genetic and Evolutionary Computation Conference. pp. 1867-1874.

[4] Assunção, W. K. G., Colanzi, T. E., Vergilio, S. R., Pozo, A., 2014. A multi-objective optimization approach for the integration and test order problem. Information Sciences 267 (0), 119-139.

[5] Auer, P., Cesa-Bianchi, N., Fischer, P., May 2002. Finite-time analysis of the multiarmed bandit problem. Machine Learning 47 (2-3), 235-256.

[6] Basgalupp, M. P., Barros, R. C., da Silva, T. S., Carvalho, A. C. P. L. F., 2013. Software Effort Prediction: A Hyper-heuristic Decision-tree Based Approach. In: ACM Symposium on Applied Computing. ACM, pp. 11091116.

s5o [7] Briand, L. C., Feng, J., Labiche, Y., 2002. Using genetic algorithms and coupling measures to devise optimal integration test orders. In: International Conference on Software Engineering and Knowledge Engineering. ACM, pp. 43-50.

[8] Burke, E. K., Gendreau, M., Hyde, M., Kendall, G., Ochoa, G., Özcan, E., $\mathrm{Qu}$, R., 2013. Hyper-heuristics: A survey of the state of the art. Journal of the Operational Research Society 64 (12), 1695-1724.

[9] Burke, E. K., Hyde, M., Kendall, G., Ochoa, G., Özcan, E., Woodward, J. R., 2010. A classification of hyper-heuristic approaches. In: Handbook of Metaheuristics. Vol. 146 of International Series in Operations Research \& Management Science. Springer US, pp. 449-468.

[10] Chakhlevitch, K., Cowling, P., 2008. Hyperheuristics: Recent developments. In: Adaptive and Multilevel Metaheuristics. Vol. 136 of Studies in Computational Intelligence. Springer Berlin Heidelberg, pp. 3-29. 
[11] Coello, C. A. C., Lamont, G. B., Veldhuizen, D. A. V., 2007. Evolution-

[16] Doerner, K., Gutjahr, W., Hartl, R., Strauss, C., Stummer, C., 2004. Pareto ant colony optimization: A metaheuristic approach to multiobjective portfolio selection. Annals of Operations Research 131 (1-4), 79-99.

[17] Durillo, J. J., Nebro, A. J., 2011. jMetal: A Java framework for multiobjective optimization. Advances in Engineering Software 42, 760-771.

[18] Fialho, A., Da Costa, L., Schoenauer, M., Sebag, M., 2009. Dynamic multiarmed bandits and extreme value-based rewards for adaptive operator selection in evolutionary algorithms. In: Learning and Intelligent Optimization. Vol. 5851 of Lecture Notes in Computer Science. Springer Berlin Heidel890 berg, pp. 176-190. 
[19] Fialho, A., da Costa, L., Schoenauer, M., Sebag, M., 2010. Analyzing bandit-based adaptive operator selection mechanisms. Annals of Mathematics and Artificial Intelligence 60 (1-2), 25-64.

[20] Furtuna, R., Curteanu, S., Leon, F., 2012. Multi-objective optimization of a stacked neural network using an evolutionary hyper-heuristic. Applied Soft Computing 12 (1), 133-144.

[21] Guizzo, G., Fritsche, G. M., Vergilio, S. R., Pozo, A. T. R., 2015. A hyperheuristic for the multi-objective integration and test order problem. In: Genetic and Evolutionary Computation Conference. ACM.

[22] Harman, M., Burke, E., Clarke, J., Yao, X., 2012. Dynamic adaptive search based software engineering. In: International Symposium on Empirical Software Engineering and Measurement. ACM.

[23] Harman, M., Mansouri, S. A., Zhang, Y., 2012. Search-based Software Engineering: Trends, Techniques and Applications. ACM Computing Surveys 45 (1), 11-61.

[24] Hartland, C., Baskiotis, N., Gelly, S., Sebag, M., Teytaud, O., 2007. Change point detection and meta-bandits for online learning in dynamic environments. In: Conférence Francophone sur l'Apprentissage Automatique. pp. $237-250$.

[25] Hashim, N., Schmidt, H., Ramakrishnan, S., Sep. 2005. Test order for classbased integration testing of Java applications. In: International Conference on Quality Software. pp. 11-18.

[26] Hewett, R., Kijsanayothin, P., Nov. 2009. Automated test order generation for software component integration testing. In: International Conference on Automated Software Engineering. pp. 211-220.

[27] Jia, Y., Cohen, M., Harman, M., Petke, J., May 2015. Learning combinatorial interaction test generation strategies using hyperheuristic search. In: International Conference on Software Engineering. 
[28] Kateb, D. E., Fouquet, F., Bourcier, J., le Traon, Y., Oct. 2014. Optimizing multi-objective evolutionary algorithms to enable quality-aware software provisioning. In: International Conference on Quality Software. pp. 85-94.

[29] Kendall, G., Soubeiga, E., Cowling, P., 2002. Choice function and random hyperheuristics. In: Asia-Pacific Conference on Simulated Evolution And Learning. Springer, pp. 667-671.

[30] Knowles, J., Corne, D., 1999. The Pareto archived evolution strategy: a new baseline algorithm for Pareto multiobjective optimisation. In: Congress on Evolutionary Computation. Vol. 1.

[31] Kumari, A. C., Srinivas, K., Gupta, M. P., Feb. 2013. Software module clustering using a hyper-heuristic based multi-objective genetic algorithm. In: Advance Computing Conference. pp. 813-818.

[32] Kung, D., Gao, J., Hsia, P., Toyoshima, Y., Chen, C., Aug. 1995. A test strategy for object-oriented programs. In: Computer Software and Applications Conference. pp. 239-244.

[33] Lai, T. L., Robbins, H., 1985. Asymptotically efficient adaptive allocation rules. Advances in Applied Mathematics 6 (1), 4-22.

[34] Li, K., Deb, K., Zhang, Q., Kwong, S., Oct. 2015. An Evolutionary ManyObjective Optimization Algorithm Based on Dominance and Decomposition. IEEE Transactions on Evolutionary Computation 19 (5), 694-716.

[35] Li, K., Fialho, A., Kwong, S., Zhang, Q., Feb. 2014. Adaptive operator selection with bandits for a multiobjective evolutionary algorithm based on decomposition. IEEE Transactions on Evolutionary Computation 18 (1), $114-130$.

[36] Maashi, M., Özcan, E., Kendall, G., 2014. A multi-objective hyper-heuristic based on choice function. Expert Systems with Applications 41 (9), 44754493. 
[37] Mariani, T., Guizzo, G., Vergilio, S. R., Pozo, A. T. R., 2016. A Grammatical Evolution Hyper-Heuristic for the Integration and Test Order Problem. In: Genetic and Evolutionary Computation Conference.

[38] Maturana, J., Fialho, A., Saubion, F., Schoenauer, M., Sebag, M., May 2009. Extreme compass and Dynamic Multi-Armed Bandits for Adaptive Operator Selection. In: Congress on Evolutionary Computation. pp. 365372.

[39] McClymont, K., Keedwell, E. C., 2011. Markov chain hyper-heuristic (MCHH): An online selective hyper-heuristic for multi-objective continuous problems. In: Conference on Genetic and Evolutionary Computation. ACM, pp. 2003-2010.

[40] Mitchell, M., 1996. An Introduction to Genetic Algorithms. MIT Press.

[41] Ochoa, G., 2013. Search methodologies in real-world software engineering. In: Conference on Genetic and Evolutionary Computation. ACM, pp. 10851088 .

[42] Rafique, A. F., 2012. Multiobjective hyper heuristic scheme for system design and optimization. In: International Conference on Mathematical Problems in Engineering, Aerospace and Sciences. Vol. 1493. pp. 764-769.

[43] Segura, C., Segredo, E., Leon, C., Feb. 2012. Analysing the adaptation level of parallel hyperheuristics applied to multiobjectivised benchmark problems. In: International Conference on Parallel, Distributed and NetworkBased Processing. pp. 138-145.

[44] Shafi, K., Bender, A., Abbass, H., 2012. Multi objective learning classifier systems based hyperheuristics for modularised fleet mix problem. In: Simulated Evolution and Learning. Vol. 7673. Springer Berlin Heidelberg, pp. 381-390. 
[45] Traon, Y., Jéron, T., Jézéquel, J.-M., Morel, P., 2000. Efficient objectoriented integration and regression testing. IEEE Transactions on Reliability $49(1), 12-25$.

975

[46] Vazquez-Rodriguez, J. A., Petrovic, S., Nov. 2013. A mixture experiments multi-objective hyper-heuristic. Journal of the Operational Research Society $64(11), 1664-1675$.

[47] Vergilio, S. R., Pozo, A., Árias, J. a. C. G., da Veiga Cabral, R., Nobre, T., 2012. Multi-objective optimization algorithms applied to the class integration and test order problem. International Journal on Software Tools for Technology Transfer 14 (4), 461-475.

[48] Wang, Z., Li, B., Wang, L., Li, Q., 2011. A brief survey on automatic integration test order generation. In: International Conference on Software Engineering and Knowledge Engineering. pp. 254-257.

985

[49] Zitzler, E., Laumanns, M., Thiele, L., 2001. SPEA2: improving the strength Pareto evolutionary algorithm. Tech. rep., Department of Electrical Engineering, Swiss Federal Institute of Technology.

[50] Zitzler, E., Thiele, L., Laumanns, M., Fonseca, C., da Fonseca, V., Apr. 2003. Performance assessment of multiobjective optimizers: an analysis and 